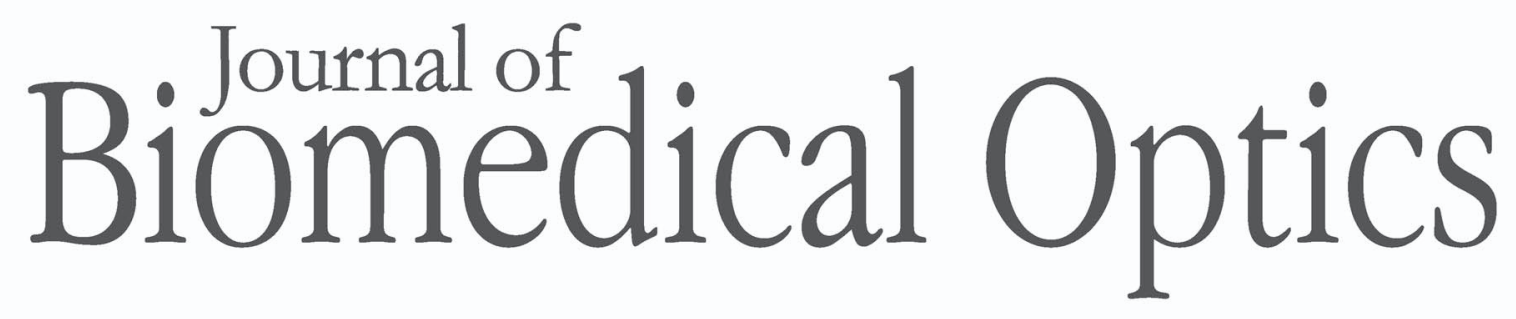

\title{
Sensitivity of Raman spectroscopy to normal patient variability
}

Elizabeth Vargis

Teresa Byrd

Quinisha Logan

Dineo Khabele

Anita Mahadevan-Jansen 


\title{
Sensitivity of Raman spectroscopy to normal patient variability
}

\author{
Elizabeth Vargis, ${ }^{\text {a }}$ Teresa Byrd, ${ }^{\mathrm{b}}$ Quinisha Logan, ${ }^{\mathrm{b}}$ Dineo Khabele, ${ }^{\mathrm{c}}$ and Anita Mahadevan-Jansen ${ }^{\mathrm{a}}$ \\ a Vanderbilt University, Department of Biomedical Engineering, VU Station Box 3515631, Nashville, Tennessee 37235 \\ ${ }^{b}$ Meharry Medical College, Department of Obstetrics and Gynecology, Nashville, Tennesee, 37208 \\ ${ }^{c}$ Vanderbilt University, Department of Obstetrics and Gynecology, Nashville, Tennessee 37235
}

\begin{abstract}
Many groups have used Raman spectroscopy for diagnosing cervical dysplasia; however, there have been few studies looking at the effect of normal physiological variations on Raman spectra. We assess four patient variables that may affect normal Raman spectra: Race/ethnicity, body mass index (BMI), parity, and socioeconomic status. Raman spectra were acquired from a diverse population of 75 patients undergoing routine screening for cervical dysplasia. Classification of Raman spectra from patients with a normal cervix is performed using sparse multinomial logistic regression (SMLR) to determine if any of these variables has a significant effect. Results suggest that BMI and parity have the greatest impact, whereas race/ethnicity and socioeconomic status have a limited effect. Incorporating BMI and obstetric history into classification algorithms may increase sensitivity and specificity rates of disease classification using Raman spectroscopy. Studies are underway to assess the effect of these variables on disease. O 2011 Society of Photo-Optical Instrumentation Engineers (SPIE). [DOI: 10.1117/1.3646210]
\end{abstract}

Keywords: Raman spectroscopy; cervical dysplasia; sparse multinomial logistic regression; fiber-optic applications.

Paper 11330PRR received Jul. 21, 2011; revised manuscript received Jun. 29, 2011; accepted for publication Sep. 13, 2011; published online Oct. 28, 2011.

\section{Introduction}

Multiple research groups have taken advantage of the extreme sensitivity of Raman spectroscopy to detect subtle changes in a variety of samples. Raman spectroscopy has been used to solve many types of problems, from detecting malignant areas in various sites, such as the cervix, ${ }^{1}$ bladder, ${ }^{2,3}$ colon,,${ }^{4,5}$ breast, ${ }^{6,7}$ and esophagus ${ }^{8,9}$ in vivo and in vitro, detecting controlled substances, ${ }^{10}$ and authenticating works of art. ${ }^{11}$ Raman spectroscopy is useful for multiple applications because it is a molecular-specific technique that provides detailed information about the biochemical composition of a sample by probing vibrational or rotational transitions in chemical bonds. Therefore, a Raman spectrum consists of a series of spectrally narrow peaks and valleys that represent the different vibrational modes of specific scattering molecules. These peaks are associated with specific bonds, such that a Raman spectrum may be referred to as a biochemical fingerprint of a molecule, tissue, or sample. Changes in peaks may be related to differences in the concentration of glycogen or collagen, ${ }^{12}$ which is useful in cancer detection, benzoic acid rings for drug detection, ${ }^{13}$ and natural or synthetic stains to decipher ages of works of art. ${ }^{14}$

Although Raman spectroscopy is inherently a sensitive technique, previous studies using Raman to detect cervical dysplasia both in vivo and in vitro have reported a wide range of sensitivity $(70-100 \%)$ and specificity $(70-100 \%)$ rates. ${ }^{1,15-19}$ Krishna et al. used a benchtop Raman spectroscopy system to acquire information from cervical samples ex vivo and classified normal compared to malignant samples at sensitivity and specificity rates of $75-99.5 \% .{ }^{19}$ Because infection with certain strains of

Address all correspondence to: Anita Mahadevan-Jansen, Department of Biomedical Engineering, Vanderbilt University, VU Station B Box 351631, Nashville, Tennessee 37235. Tel: 615-343-4787; E-mail: anita.mahadevanjansen@vanderbilt.edu. human papillomavirus (HPV) is the cause of cervical dysplasia in $>99 \%$ of cases worldwide, ${ }^{20-22}$ certain research groups have used Raman spectroscopy to identify differences between HPV types. Jess et al. used a confocal Raman microscope system and were able to discriminate varying HPV types in live and fixed cells with sensitivity and specificity rates of $70-100 \% .{ }^{15}$ Our research group has used a portable probe-based Raman spectroscopy system to distinguish between normal and malignant cervical samples in cell culture, in vitro and in vivo. The sensitivity and specificity rates of this work have ranged between 81 and $97 \% .^{1,16,17}$

Variations in classification rates that are observed when using Raman spectroscopy to detect malignant areas of the cervix are a hurdle that must be overcome in order for this technology to benefit clinical practice. Fortunately, as more research into using Raman spectroscopy to diagnose cervical dysplasia has been conducted, it has become apparent that by accounting for normal patient variations, the sensitivity and specificity of Raman for diagnosing disease can increase. ${ }^{23,24}$ Similar research on inherent normal patient variability has been seen when using Raman on other tissue sites, such as the colon and the breast. ${ }^{25-27}$ In many cases, accounting for these normal differences has led to an increase in both the sensitivity and specificity rates of classifying normal spectra compared to disease.

Specifically, research from our lab has shown that both hormonal differences due to menstrual cycles and menopause $\mathrm{e}^{23}$ and previous disease or presence near disease ${ }^{24}$ have a significant impact on the Raman spectra acquired from benign areas of the cervix in vivo as well as the classification of spectra acquired from metaplastic and dysplastic sites. During a menstrual cycle and menopause, varying levels of hormones are released throughout the body that can cause changes in the cervix,

1083-3668/2011/16(11)/117004/9/\$25.00 @ 2011 SPIE 
including softening, drying, or thinning. ${ }^{28,29}$ Permanent field effects or malignancy-associated changes that result from the presence or prior history of disease may account for the significant effect of such differences on Raman spectra. ${ }^{30,31}$ Accounting for hormonal levels and history of disease prior to disease classification resulted in sensitivity and specificity rates of $>95 \%$. By recognizing and accounting for these normal variations prior to disease classification, better training sets were used for the classification algorithms and, therefore, differences among spectra were more likely to result from malignancy than normal variations. Other factors may further improve cervical disease classification with Raman spectroscopy.

Race and ethnicity, for example, as well as socioeconomic status are all correlated with different incidences of cervical malignancies. ${ }^{32}$ In 2010, black and Hispanic populations had the highest incidence of and mortality rates from cervical dysplasia in the U.S. (11.1 cases and 4.6 deaths and 12.7 cases and 3.1 deaths per 100,000 women, respectively, compared to 7.9 cases and 2.2 deaths per 100,000 white women). ${ }^{33,34}$ Worldwide, cervical cancer is the fifth most common cancer for women and the most common cancer for women in Central America and southern Africa. ${ }^{35}$ These differences are likely correlated with reduced access to routine care and screening. Obesity is also associated with higher rates of cancer, including cervical cancer. $^{22,36,37}$ There are many potential reasons for the contribution of obesity to cervical cancer, including differences in vaginal flora patterns, steroid hormone and cholesterol levels, cultural norms, and bacterial or viral infections. ${ }^{36}$ HPV could also be a potential factor in the differences in incidence and mortality rates because several at-risk strains are more prevalent in certain racial and ethnic populations. ${ }^{20-22}$ Any of these characteristics may significantly influence the biochemical makeup of the cervix and, thus, the Raman spectra acquired from the cervix. Such important variables must be accounted for prior to disease classification to increase classification accuracy rates.

It is interesting to explore whether other optical techniques are similarly influenced by normal patient variables. Studies have shown that fluorescence spectroscopy is not significantly influenced by changes that occur during a woman's menstrual cycle or menopause because it only causes a variation of 8 $16 \%$ in normal spectra. ${ }^{38,39}$ This result suggests that fluorescence spectroscopy is not sensitive to the small biochemical changes that occur as a result of the fluctuations in normal hormonal levels. Biochemical changes due to hormonal fluctuations, age, and parity have been observed in the breast using reflectance and transmittance spectroscopy ${ }^{40,41}$ however, their effect on disease classification has yet to be determined. Similar results have been found in the cervix, ${ }^{42}$ but these results are controlled by levels of hemoglobin, water, and lipids, instead of the broader biochemical fingerprint obtained with Raman spectroscopy. Kelly et al. used infrared (IR) spectroscopy to separate samples based on HPV infection and age and were able to show separation of samples using principal component analysis followed by linear discriminant analysis, but this in vitro study was performed on cells grown in culture. ${ }^{43}$ IR spectroscopy, analogous to Raman spectroscopy, may perform with similar sensitivity rates. However, the water content found in bulk tissue may inhibit IR spectra, thereby reducing its utility as a tool for in vivo detection. To our knowledge, there are no published reports that consider the effect of race/ethnicity, body mass in- dex (BMI), obstetric history, or socioeconomic status on optical measurements.

The goal of this study is to examine the significance of such normal variations on the classification of Raman spectra. Implementing Raman spectroscopy in a clinical setting will require it to be successfully applied to any patient population, regardless of race or ethnicity, BMI, parity, or socioeconomic status. To accomplish this goal, Raman measurements were acquired from a diverse patient population without current or previous disease. A classification algorithm, sparse multinomial logistic regression (SMLR), was then used to determine if the Raman spectra were significantly affected by race/ethnicity, BMI, obstetric history, or socioeconomic status.

\section{Materials and Methods}

In our lab, previous studies were performed on a predominantly white population with BMI levels of normal to overweight. 1,17,23,24 For this study, patients were recruited from the county hospital in Nashville, Tennessee (Nashville General Hospital at Meharry) to acquire data from patients of varying racial/ethnic background, BMI, obstetric history, and socioeconomic status. This study was approved by the Meharry Medical College Institutional Review Board.

\subsection{Patient Enrollment}

A total of 75 adult female patients undergoing a routine Pap smear consented to participate in the study. The patient's age, date of last menstrual period, use of artificial hormones, menopausal status, height, weight, obstetric history, ethnicity, address, insurance, relevant medical history, and any previous abnormal Pap smears were all noted upon chart review. After the cervix was exposed and visually examined by the attending physician, the cervix was wiped clean with a dry cotton swab followed by saline. Raman measurements were then taken from three locations on the ectocervix. Next, the Pap smear procedure was done according to standard clinical protocol. The spectra were correlated with histological results and considered normal if the Pap smear was negative.

\subsection{Patient Information}

Patients were stratified according to four sets of data to determine the sensitivity of Raman spectroscopy: (i) Race/ethnicity (white, black, and Hispanic), (ii) BMI category (normal, overweight, and obese), (iii) parity (no pregnancies or $\geq 1$ ), and (iv) socioeconomic status (uninsured or insured, determined by whether the patient had government-subsidized or private health insurance). Racial or ethnic group was determined by what the patient identified herself as. BMI was calculated (using the following formula) from the height and weight measured the same day when Raman spectra were acquired: ${ }^{44}$

$$
\mathrm{BMI}=\frac{\operatorname{mass}(\mathrm{lb}) \times 703}{[\text { height }(\mathrm{in})]^{2}} .
$$

BMI values that define specific categories used in this study-normal, overweight, and obese-are presented in Table 1. Obstetric history was determined from a chart review. 
Table 1 Weight status based on body mass index (BMI).

\begin{tabular}{lc}
\hline Weight category & $\mathrm{BMl}\left(\mathrm{kg} / \mathrm{m}^{2}\right)$ \\
\hline Normal & $18.50-24.99$ \\
Overweight & $25.00-29.99$ \\
Obese & $\geq 30.00$ \\
\hline
\end{tabular}

\subsection{Instrumentation and Data Processing}

Raman spectra were collected in vivo using a portable Raman spectroscopy system, consisting of a 785-nm diode laser (PI-ECL-785-350, Process Instruments, Inc., Salt Lake City, Utah), a beam-steered fiber-optic probe (Visionex, Atlanta, Georgia), an imaging spectrograph (Holospec f/1.8i-NIR, Kaiser Optical Systems, Ann Arbor, Michigan), and a back-illuminated, deep-depletion, thermoelectrically cooled CCD camera (Pixis 256BR, Princeton Instruments, Princeton, New Jersey), all controlled with a laptop computer. Details of the system have been reported previously. ${ }^{45}$ The fiber-optic probe delivered $80 \mathrm{~mW}$ of incident light onto the tissue at an integration time of $2-3 \mathrm{~s}$, with all room lights and the computer monitor turned off. The system provided a spectral resolution of eight wavenumbers $\left(\mathrm{cm}^{-1}\right)$.

Spectral calibration of the system was performed each day using a neon-argon lamp and naphthalene and acetaminophen standards to correct for day to day variations. A National Institute of Standards and Technology (NIST)-calibrated tungsten lamp was also used to account for the wavelength-dependent response of the system. The spectra were processed for fluorescence subtraction and noise smoothing using the modified polynomial fit and Savitzy-Golay methods, described previously. ${ }^{45}$ Following data processing, each spectrum was normalized to its mean spectral intensity across all Raman bands to account for intensity variability.

\subsection{Data Analysis}

As previously reported, menopausal status and history of cervical disease affects Raman spectra. ${ }^{23}$ For this reason, only premenopausal patients with no history of cervical disease were used in this analysis. A composite spectrum averaging Raman measurements from each patient was used for analysis because it would contain the effects of any significant patient variations. Discrimination was performed with SMLR, a Bayesian machine-learning framework that computes the posterior probability of a spectrum belonging to each tissue class based on a labeled training set. ${ }^{24,46}$ For this analysis, whichever class had the higher probability of membership was the one to which the spectrum was classified. Because only one composite spectrum per patient was used, SMLR was run with leave-one-patientout cross-validation. A range of input parameters to SMLR have been tested previously, and these tests revealed that the combination of parameters that provide the most accurate classification, while also maximizing sparsity, was using a Laplacian prior, direct kernel, $\lambda$ value of 0.01 , with no additional bias term.
Table 2 Patient categories. Total in all categories is 75 .

\begin{tabular}{lc} 
Patient categories & No. in category \\
\hline White & $23(31 \%)$ \\
Black & $31(39 \%)$ \\
Hispanic & $20(27 \%)$ \\
Arabic & $1(1 \%)$ \\
Underweight BMI & $2(3 \%)$ \\
Normal BMI & $21(28 \%)$ \\
Overweight BMI & $24(32 \%$ \\
Obese BMI & $28(37 \%)$ \\
No previous pregnancies & $33(44 \%)$ \\
Prior Pregnancy(ies) & $42(56 \%)$ \\
Insured & $44(59 \%)$ \\
Not Insured & $31(41 \%)$ \\
\hline
\end{tabular}

\section{Results}

The epidemiologic makeup (race/ethnicity groups, BMI category, obstetric history, and socioeconomic status) of the entire cohort of 75 patients recruited to this study is shown in Table 2. In the analyses that follow, only Raman spectra from premenopausal women with no history or current presence of cervical disease were used. Because of lower recruitment numbers, patients of Arabic descent or underweight BMI were excluded. Some spectra could not be classified into appropriate categories, such as those from women with spontaneous abortions and from women whose health insurance was only acquired recently. Those spectra were also excluded.

\subsection{Race and Ethnicity}

Raman spectra ( $n=193)$ were acquired from the cervix of patients from 3 different racial and ethnic groups (white $n=21$, black $n=23$, Hispanic $n=18$, total $=62$, excluded $=13$ ). There are many similarities across these spectra throughout the wavenumber range [Fig. 1(a)]. Figure 1(b) shows difference spectra of Raman measurements from white and black patients and white and Hispanic patients. A few of the small differences between the spectra are shown as the box plots in Figs. 1(c)-1(e), including the peaks that have been assigned in previous studies as lipid and DNA of $\sim 1303 \mathrm{~cm}^{-1}$, the phenylalanine and DNA region around $1510-1520 \mathrm{~cm}^{-1}$ and the shoulders of the $1656 \mathrm{~cm}^{-1}$ amide I peak. ${ }^{47-49}$ Changes in the shoulders of the amide I peak are usually due to changes in the secondary structures of amide I, such as the $\beta$ pleated sheet proteins. ${ }^{49}$ These peaks that seemed to qualitatively have the most difference in the averaged composite spectra have large areas of overlap. Box plots were used to visualize the data because they provide more detailed information compared to bar graphs. These figures have been explained in detail previously. ${ }^{50}$ Generally, the box 


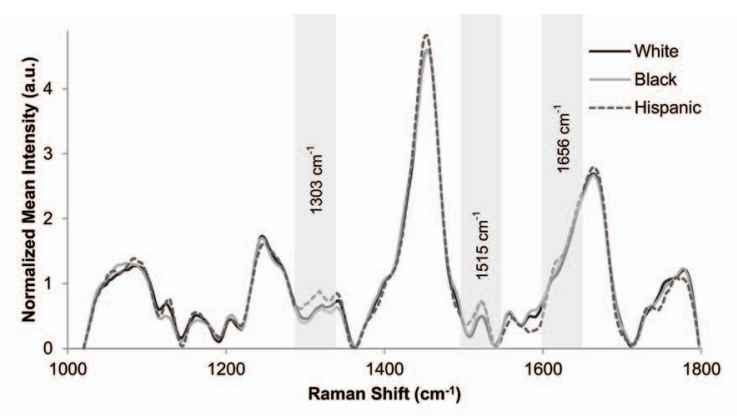

(a)

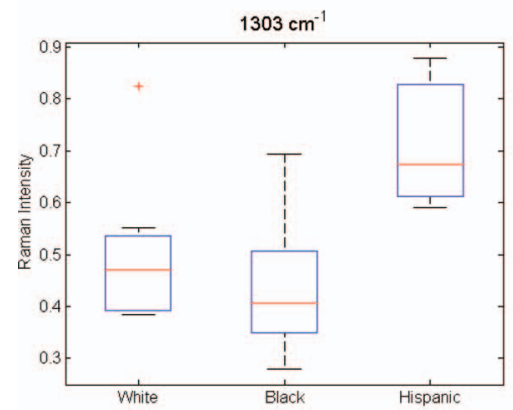

(c)

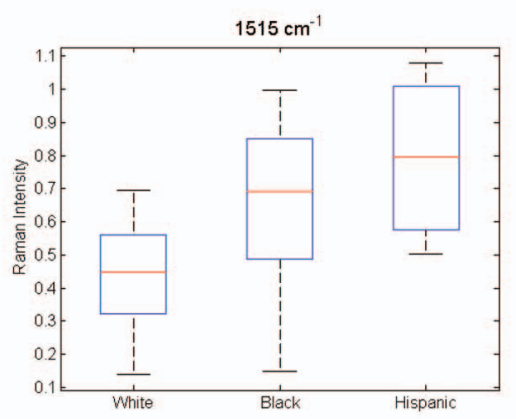

(d)

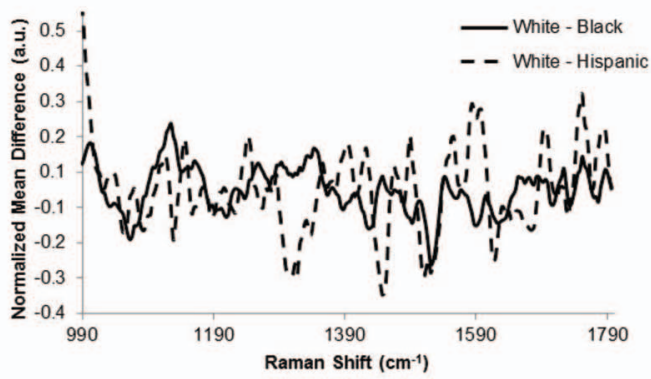

(b)

Fig. 1 (a) Normalized average Raman spectra of white, black, and Hispanic patients. Highlighted regions are displayed in (c-e). (b) Difference spectra between measurements from white and black patients and white and Hispanic patients. (c-e) Box plots of specific peaks of Raman spectra from normal cervix of patients who described themselves as white, black, or Hispanic. Potential peak assignments: (c) Lipid and DNA, (d) phenylalanine and DNA, (e) Amide I shoulder. The box contains data between the 25th and 75th percentile, with the centerline representing the median. The error bars are \pm 1 S.D. about the mean. Outliers are represented by + .

represents the 25 th and 75 th percentiles of the data as the bottom and top of the box. The centerline is the median, the error bars represent one standard deviation from the mean, and any outliers are represented by a plus sign.

\subsection{Body Mass Index}

Raman measurements $(n=187)$ from three different BMI categories (normal $n=19$, overweight $n=21$, obese $n=23$, total $=63$, excluded $=12$ ) were acquired and averaged. The spectra among these three categories appear similar in most areas, but a few significant differences occur in the peaks at 1010, 1656, and $1750 \mathrm{~cm}^{-1}$ [Fig. 2(a)]. Difference spectra have been plotted in Fig. 2(b), showing differences between patients of normal BMI and overweight BMI, as well as normal and obese. To visualize important areas of the spectra, box plots for these peaks, which have been assigned by other researchers as phenylalanine, lipid, and $\mathrm{C}=\mathrm{C}$ bonds, respectively, are shown in Figs. 2(c)-2(e). ${ }^{49,51,52}$

\subsection{Parity}

Raman measurements $(\mathrm{n}=117)$ were obtained from two groups of patients, nulliparous $(n=23)$ and parous $(n=24$, total $=47$, excluded $=28$ ). Compared to previous spectra, there are slight variations throughout the entire range [Fig. 3(a)]. A subtraction spectrum showing the differences between measurements from women who have and have not been pregnant is shown in Fig. 3(b). More significant differences are seen in the region of $1050-1130 \mathrm{~cm}^{-1}$, which have been attributed to $\mathrm{C}-\mathrm{C}$ and $\mathrm{C}-\mathrm{O}$ stretches, as well as varying concentrations of collagen, elastin, lipid, and proline. ${ }^{49,53,54}$ Box plot peaks corresponding to some of these differing regions are shown in Figs. 3(c)-3(e).

\subsection{Socioeconomic Status}

For this study, patients' health insurance status was used as an indicator of socioeconomic status. Raman spectra $(n=123)$ were obtained from the cervix of patients with or without private health insurance with 26 patients in each category [excluded $=23$; Fig. 4(a)]. Included in the group of patients without health insurance were women who qualified for the Tennessee Breast and Cervical Cancer Screening program. These patients have health care for mammograms, Pap smears, etc., but the program does not provide comprehensive health-care coverage. The spectra from the two groups mostly overlap, with only a few areas of small differences between patients with and without health insurance. This outcome is shown in Fig. 4(b). Figures 4(c)-4(e) contain box plots for the peaks at 1095, 1265, and $1656 \mathrm{~cm}^{-1}$, which correspond to $\mathrm{PO}_{2}^{-1}$, protein content, and the shoulder of the amide I band. ${ }^{49,54-56}$

\subsection{Statistical Analyses}

Although box plots have been used to highlight various regions of the spectrum, the entire normalized spectrum from 990 to $1800 \mathrm{~cm}^{-1}$ for each patient within each category was used for this analysis. The first step of this analysis was to classify the spectra according to the previous categories (i.e., white, 


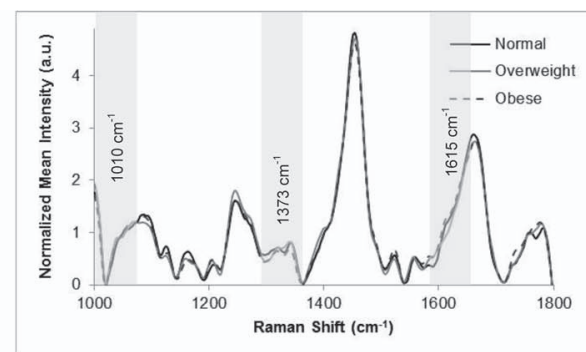

(a)

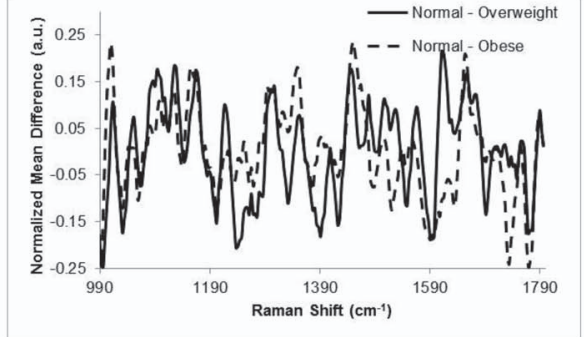

(b)

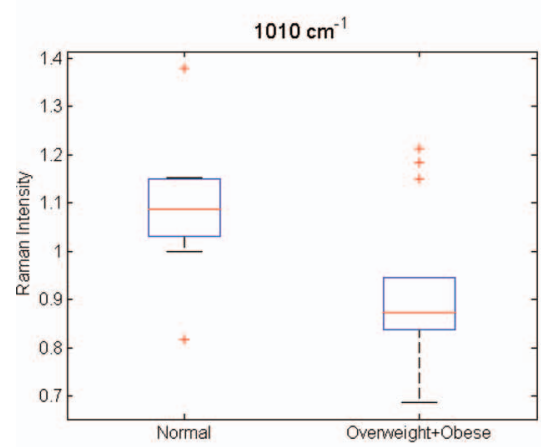

(c)

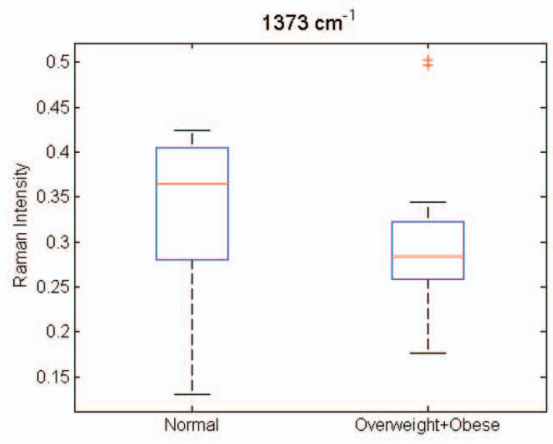

(d)

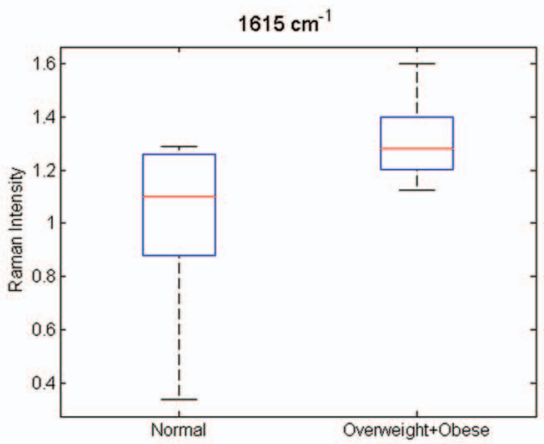

(e)

Fig. 2 (a) Normalized average Raman spectra from normal, overweight and obese patients. Highlighted regions are displayed in (c-e). (b) Difference spectra between measurements from normal and overweight patients and normal and obese patients. (c-e) Box plots showing regions of difference between patients with normal and overweight + obese BMI levels. Potential peak assignments: (c) phenylalanine, (d) lipid, (e) C = C bond. The box contains data between the 25th and 75th percentile, with the centerline representing the median. The error bars are \pm 1 S.D. about the mean. Outliers are represented by + .

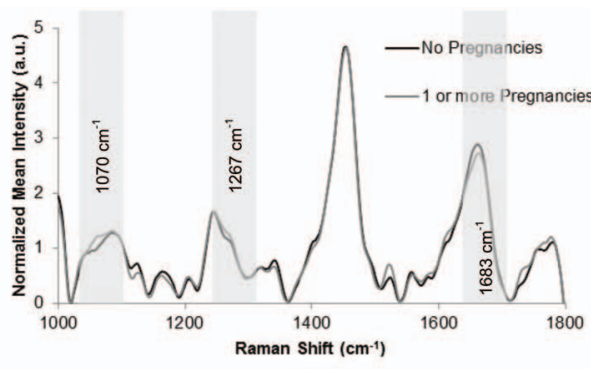

(a)

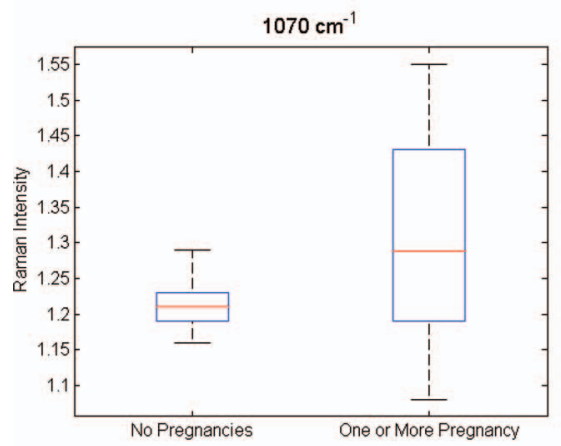

(c)

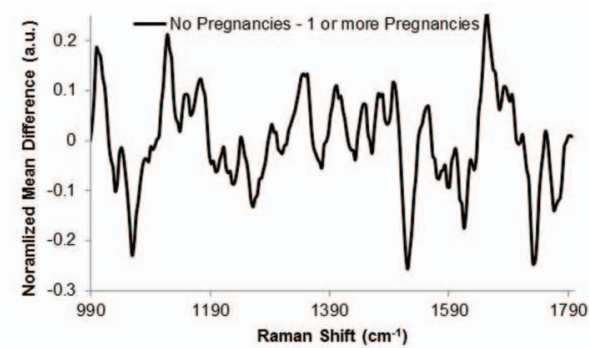

(b)

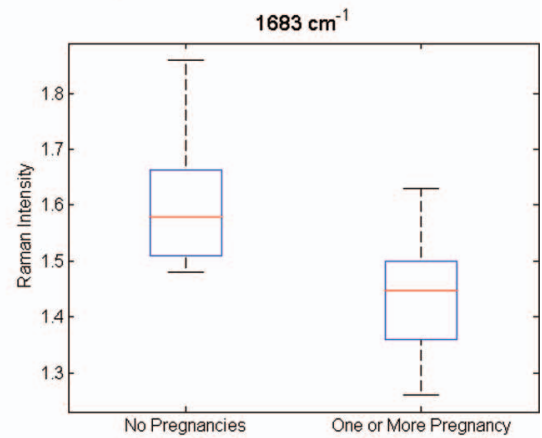

(e)

Fig. 3 (a) Normalized average Raman spectra from patients with zero pregnancies and one or more pregnancy. Highlighted regions are displayed in (c-e). (b) Difference spectra between measurements from patients with and without previous pregnancy. (c-e) Box plots showing regions of difference between patients with and without previous pregnancy. Potential peak assignments: (c) collagen/elastin, (d) Amide III, (e) C-O stretch. The box contains data between the 25 th and 75 th percentile, with the centerline representing the median. The error bars are \pm 1 S.D. about the mean. Outliers are represented by + . 


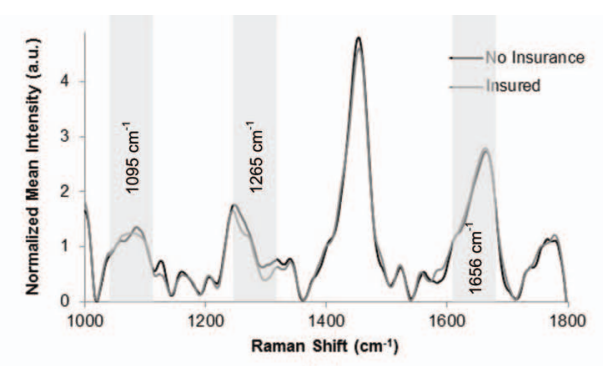

(a)

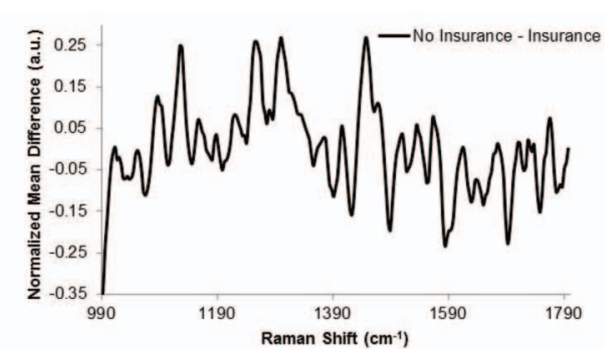

(b)

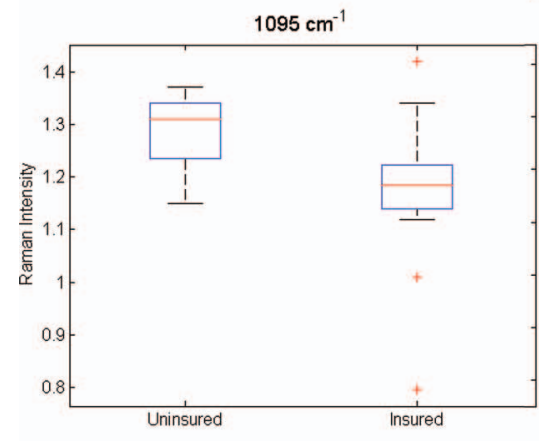

(c)

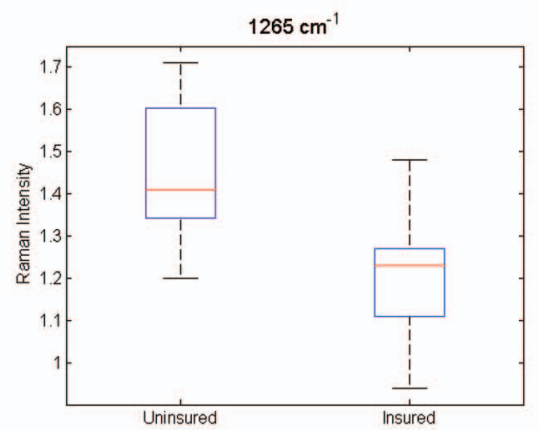

(d)

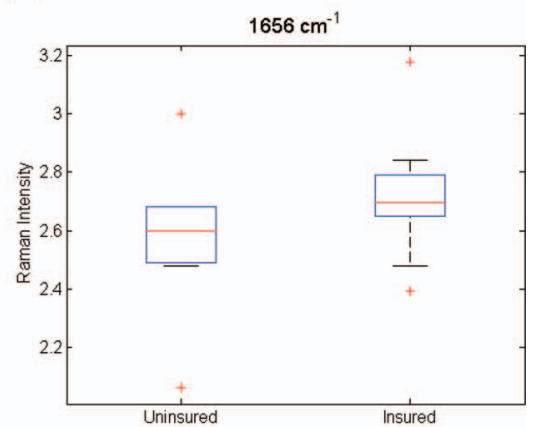

(e)

Fig. 4 (a) Normalized average Raman spectra from patients with and without health insurance. Highlighted regions are shown in (c-e). (b) Difference spectra between measurements from patients with and without health insurance. (c-e) Box plots showing regions of difference between patients with and without health insurance. Potential peak assignments: (c) $\mathrm{PO}_{2}^{-1}$, (d) protein content, (e) Amide I shoulder. The box contains data between the 25th and 75th percentile, with the centerline representing the median. The error bars are \pm 1 S.D. about the mean. Outliers are represented by + .

black, and Hispanic, etc.) to determine if significant differences exist in the Raman spectra acquired from these specific groups of patients. Various iterations of analyses were used to decide how the spectra should be classified, which was determined by finding the greatest classification accuracy of these iterations. For example, for the BMI category, SMLR was first performed to classify the spectra as normal, overweight, or obese. However, a higher classification accuracy rate was found when SMLR was performed with only two categories: normal versus a combined category of spectra from overweight and obese patients. Table 3 shows the maximum classification accuracy obtained within each patient variability category. The category of race/ethnicity classified with a maximum rate of $58 \%, \mathrm{BMI}$ with a maximum rate of $78 \%$ when spectra were classified as normal or a combined category of overweight or obese, parity with a rate of $75 \%$, and socioeconomic status with a rate of $61 \%$.

\section{Discussion}

Previous studies using Raman spectroscopy to detect cervical dysplasia have shown limited success. ${ }^{1,15,16}$ We have previously demonstrated that some of these limitations can be overcome by accounting for both normal patient variability, such as hormonal differences and the history or presence of disease. ${ }^{1,23,24}$ In this paper, we evaluate whether Raman spectra acquired from normal tissue can be separated based on other patient variables, including race/ethnicity, BMI, obstetric history, and socioeconomic status.

The spectra used in the analyses presented here were acquired from patients with no history of disease and whose current Pap smear result was within normal limits. When a pathologist examined these cytology specimens, few variations were observed and the clinical diagnosis of each patient was normal. If there are no significant differences due to common patient variations, such as ethnicity, height, and weight, then we expect spectra acquired from the cervix of patients with a normal pathology to also appear similar and have few variations. Furthermore, if these variables have no impact on the spectra, then statistical classification should produce results with accuracy rates of $\sim 50 \%$, similar to a coin toss. Previous studies have suggested the patient variables analyzed in this paper may be correlated with higher incidences of cervical dysplasia. ${ }^{32,35} \mathrm{Al}$ though we do not expect a significant impact from these normal physiological and social variables on the biochemical makeup of the cervix or the acquired Raman spectra, investigating the

Table 3 Results from statistical analyses of patient variables.

\begin{tabular}{|c|c|c|}
\hline Patient variable & $\begin{array}{l}\text { Optimized classification } \\
\text { categories (number of } \\
\text { categories used) }\end{array}$ & $\begin{array}{l}\text { Maximum } \\
\text { classification } \\
\text { accuracy }(\%\end{array}$ \\
\hline Race/ethnicity & White, black, Hispanic (3) & 58 \\
\hline BMI & $\begin{array}{c}\text { Normal, overweight }+ \text { obese } \\
\text { (2) }\end{array}$ & 78 \\
\hline Obstetric history & $\begin{array}{c}\text { No pregnancies, }, \geq 1 \\
\text { pregnancy (2) }\end{array}$ & 75 \\
\hline $\begin{array}{l}\text { Socioeconomic } \\
\text { status }\end{array}$ & Uninsured, insured (2) & 61 \\
\hline
\end{tabular}


influence of these variables is an important first step in validating the application of Raman spectroscopy on a diverse patient population. The results from this study show that some of these variables produce significant changes to Raman spectra that are separable using statistical classification methods.

The two patient variables that resulted in the most separable spectra were BMI and parity, with classification rates of 78 and $75 \%$, respectively. These results suggest that BMI and parity cause significant changes in the cervix and therefore affect any spectra acquired from the cervix. As discussed previously, higher BMI is associated with increased areas of inflammation and higher blood serum hormonal levels. ${ }^{22,24,37}$ Although there are cycling levels of hormones that affect the elasticity and softness of the cervix, varying baseline levels of steroid hormones due to BMI differences may change the cervix as well. Similarly, it is reasonable to believe that parity would influence normal Raman spectra. The cervix is known to change dramatically during pregnancy and labor. ${ }^{57}$ These changes appear to be long term and affect tissue biochemistry significantly enough to be recognizable with Raman spectroscopy. Furthermore, accuracy rates may increase by defining narrower classification groups. Figure 3(c) shows a box plot of the peak at $1070 \mathrm{~cm}^{-1}$, correlating to collagen and elastin content. Although there is little variance in patients who have not been pregnant, there is a significant amount of variance in the spectra from those who have. This variance may be correlated with number of pregnancies and type of delivery (vaginal or caesarean), both of which may change the concentration and organization of collagen. ${ }^{58}$ In the future, comparing groups of patients to different delivery types and number of pregnancies may increase accuracy rates.

Classification by socioeconomic status and race/ethnicity resulted in accuracy rates of 61 and 58\%, respectively, suggesting that these variables have a smaller impact on the Raman spectra. Although there are small differences in the Raman spectra that lead to a classification rate of $>50 \%$, socioeconomic status and race/ethnicity are less significant compared to BMI and parity. One possible explanation for the $61 \%$ classification accuracy with socioeconomic status may be an increased prevalence of undiagnosed medical problems due to the lack of medical insurance reducing access to comprehensive health care. These results also suggest that the different incidence rates of cervical dysplasia among racial and ethnic groups are not due to fundamental differences in the biochemical makeup of the cervix. Even though there is no physiological evidence that race or ethnicity change the biochemical makeup of the cervix, it was important to investigate these variables to ensure that Raman spectroscopy can be applied to detect cervical dysplasia in diverse populations. The low rate of classifying data by race or ethnicity alone suggests that these variations do not significantly affect the cervix or the classification of Raman spectra. This outcome is significant because it indicates Raman spectroscopy may be applied in clinical settings on any patient population.

The success of the analyses performed in this study depends on the reliability of the data used to categorize the spectra. For example, the patient reported her own race or ethnicity, but the categories were limited to "white," "black," and "Hispanic," and she may fit into more than one or none of these. BMI is a controversial measure of body fat that may be inaccurate because it does not reflect changes due to age, race, gender, etc. ${ }^{59}$ To classify spectra based on parity, higher rates may be achieved by further categorizing previously pregnant patients by number of pregnancies and number and type of delivery (vaginal or cesarean). In this study, some spectra $(n=11)$ were acquired during a patient's postpartum exam, typically 6-10 weeks after delivery. Obtaining data at this point may not only affect spectra acquired from the cervix, but also a patient's BMI. Future studies on patient variability should exclude data from such patients. Finally, whether or not a patient has health-care coverage may not be an accurate measure of socioeconomic status or other cultural practices, such as dietary and social habits that are associated with different economic levels. To truly investigate the influence of specific variables, surveys of the patients may be more beneficial.

Studies from our lab and others have looked at the effect of hormonal variations,${ }^{28}$ age,${ }^{26}$ acetic acid,${ }^{24}$ creams and lotions, ${ }^{27}$ history or presence of disease, ${ }^{24,25}$ smoking, BMI, obstetric history, insurance status, and race/ethnicity on Raman spectra obtained from nonmalignant areas of the cervix and other tissues. The results from previous work have shown that, prior to classification, stratifying spectra based on hormonal changes and history or presence of disease reduces intraclass variability among both normal and diseased spectra. ${ }^{1,24}$ Unsupervised classification algorithms were then more likely to account for the variance due to differences from disease instead of the variance due hormonal status or disease history, resulting in improved performance. However, close examination of these results revealed that a significant amount of intraclass variance among the normal spectra remained, ${ }^{1,23,24}$ which motivated investigating additional factors that could be used to account for the variance among normal spectra and improve classification of disease.

Therefore, the purpose of this paper is to perform the critical intermediate step of identifying the most separable sources of intraclass variation among normal spectra. Our results indicate that of the variables investigated, normal spectra are best separated as a function of BMI and parity, which are both easily obtainable from the patient. On the basis of previous experience with hormonal status and disease history, it is possible that stratification of the spectra by BMI and parity may also improve the accuracy of disease diagnosis using Raman spectroscopy. Because an effect of race/ethnicity or socioeconomic status on classification was not identified, there appears to be no benefit to accounting for those variables prior to classification. Consideration of other variables beyond those discussed here may be important and can be determined based on organ sites. However, the cost to benefit ratio of incorporating additional physiological patient variables prior to spectral classification must be considered before further analysis is performed.

Current research is underway in our lab to understand the significance of BMI, parity, race/ethnicity, and socioeconomic status on disease classification and to quantify the relative variance these factors impart on normal spectra compared to diseased spectra. Although it may be possible that BMI and parity do not affect disease spectra as has been demonstrated with normal spectra, they are still important factors to be aware of prior to classification of cervical dysplasia.

The goal of using Raman spectroscopy for precancer detection is to provide an automated, real-time method of accurately detecting malignant cells in any patient population, regardless 
of race, ethnicity, BMI, parity, or socioeconomic status. Previous work using Raman spectroscopy for in vivo screening and diagnosis of cervical dysplasia has shown promising results, with sensitivities and specificities of $>90 \%$; however, this work was done in a fairly homogenous patient population. In this study, we investigated the spectral variations in a more diverse patient group and found more distinct differences due to BMI and obstetric history compared to race/ethnicity and socioeconomic status. We believe these results suggest that classification algorithms for the detection of cervical dysplasia with Raman spectroscopy should be developed to incorporate BMI and obstetric history, but it does not appear necessary to control for race, ethnicity, or a patient's socioeconomic status. These results also suggest that normal sources of physiological variability, such as hormone levels, BMI, etc., may affect the inherent baseline Raman spectra acquired from other organ systems as well. For example, skin pigment and dryness may be important variables to account for when using Raman spectroscopy for diagnosing melanoma. Age and gender may be significant factors that impact the biochemical makeup of the GI tract. Parity and hormonal fluctuations may affect the classification accuracy of Raman spectra acquired from the ovary. By examining the effect of such normal physiological variables on spectra, the sensitivity of Raman spectroscopy will be revealed, as will its effectiveness for detecting and diagnosing disease.

\section{Acknowledgments}

The authors acknowledge the financial support of the National Institute of Health Grant No. R01-CA-095405 and a predoctoral fellowship (Grant No. T32-HL7751-15) for E.V. Special thanks go to the nurses and staff at Meharry Medical College for their help, and to Chetan Patil and Amy Rudin for proofreading this paper.

\section{References}

1. E. M. Kanter, E. Vargis, S. Majumder, M. D. Keller, E. Woeste, G. G. Rao, and A. Mahadevan-Jansen, "Application of Raman spectroscopy for cervical dysplasia diagnosis," J. Biophoton. 2, 81-90 (2009).

2. P. Crow, A. Molckovsky, N. Stone, J. Uff, B. Wilson, and L. M. WongKeeSong, "Assessment of fiberoptic near-infrared raman spectroscopy for diagnosis of bladder and prostate cancer," Urology 65, 1126-1130 (2005).

3. M. C. Grimbergen, C. F. van Swol, R. J. van Moorselaar, J. Uff, A. Mahadevan-Jansen, and N. Stone, "Raman spectroscopy of bladder tissue in the presence of 5-aminolevulinic acid," J. Photochem. Photobiol. $B$ 95, 170-176 (2009).

4. C. Krafft, A. A. Ramoji, C. Bielecki, N. Vogler, T. Meyer, D. Akimov, P. Rosch, M. Schmitt, B. Dietzek, I. Petersen, A. Stallmach, and J. Popp, "A comparative Raman and CARS imaging study of colon tissue," $J$. Biophoton. 2, 303-312 (2009).

5. M. V. Chowdary, K. K. Kumar, K. Thakur, A. Anand, J. Kurien, C. M. Krishna, and S. Mathew, "Discrimination of normal and malignant mucosal tissues of the colon by Raman spectroscopy," Photomed. Laser Surg. 25, 269-274 (2007).

6. M. V. Chowdary, K. Kalyan Kumar, S. Mathew, L. Rao, C. M. Krishna, and J. Kurien, "Biochemical correlation of Raman spectra of normal, benign and malignant breast tissues: a spectral deconvolution study," Biopolymers 91, 539-546 (2009).

7. S. K. Majumder, M. D. Keller, F. I. Boulos, M. C. Kelley, and A. Mahadevan-Jansen, "Comparison of autofluorescence, diffuse reflectance, and Raman spectroscopy for breast tissue discrimination," $J$. Biomed. Opt. 13, 054009 (2008).
8. H. Barr, C. Kendall, F. Bazant-Hegemark, P. Moayyedi, G. Shetty, and N. Stone, "Endoscopic screening and surveillance for Barrett's esophagus—clinical implications," MedGenMed 8(2), 88 (2006).

9. G. Shetty, C. Kendall, N. Shepherd, N. Stone, and H. Barr, "Raman spectroscopy: elucidation of biochemical changes in carcinogenesis of oesophagus," Br. J. Cancer 94, 1460-1464 (2006).

10. C. Eliasson, N. A. Macleod, and P. Matousek, "Non-invasive detection of cocaine dissolved in beverages using displaced Raman spectroscopy," Anal. Chim. Acta 607, 50-53 (2008).

11. M. Leona, P. Decuzzi, T. A. Kubic, G. Gates, and J. R. Lombardi, "Nondestructive identification of natural and synthetic organic colorants in works of art by surface enhanced Raman scattering," Anal. Chem. (2011).

12. V. Kumar and S. L. Robbins, Robbins Basic Pathology, Saunders/Elsevier, Philadelphia, (2007).

13. A. P. Gamot, G. Vergoten, and G. Fleury, "Etude par spectroscopie raman du chlorhydrate de cocaine," Talanta 32, 363-372 (1985).

14. R. L. Feller, A. Roy, E. W. FitzHugh, and B. H. Berrie, Artists' Pigments: A Handbook of Their History and Characteristics, National Gallery of Art, Washington (1986).

15. P. R. T. Jess, D. D. W. Smith, M. Mazilu, K. Dholakia, A. Riches, and C. S. Herrington, "Early detection of cervical neoplasia by Raman spectroscopy," Int. J. Cancer 121, 2723-2728 (2007).

16. A. Mahadevan-Jansen, M. F. Mitchell, N. Ramanujam, A. Malpica, S. Thomsen, U. Utzinger, and R. Richards-Kortum, "Near-infrared Raman spectroscopy for in vitro detection of cervical precancers," Photochem. Photobiol. 68, 123-132 (1998).

17. A. Robichaux-Viehoever, E. Kanter, H. Shappell, D. Billheimer, H. Jones, III, and A. Mahadevan-Jansen, "Characterization of Raman spectra measured in vivo for the detection of cervical dysplasia," Appl. Spectrosc. 61, 986-993 (2007).

18. Y. Yazdi, N. Ramanujam, R. Lotan, M. F. Mitchell, W. Hittelman, and R. Richards-Kortum, "Resonance Raman spectroscopy at $257 \mathrm{~nm}$ excitation of normal and malignant cultured breast and cervical cells," Appl. Spectrosc. 53, 82-85 (1999).

19. C. M. Krishna, N. B. Prathima, R. Malini, B. M. Vadhiraja, R. A. Bhatt, D. J. Fernandes, P. Kushtagi, M. S. Vidyasagar, and V. B. Kartha, "Raman spectroscopy studies for diagnosis of cancers in human uterine cervix," Vib. Spectrosc. 41, 136-141 (2006).

20. J. M. M. Walboomers, M. V. Jacobs, M. M. Manos, F. X. Bosch, J. A. Kummer, K. V. Shah, P. J. F. Snijders, J. Peto, C. J. L. M. Meijer, and N. Muñoz, "Human papillomavirus is a necessary cause of invasive cervical cancer worldwide," J. Pathol. 189, 12-19 (1999).

21. E. F. Dunne, E. R. Unger, M. Sternberg, G. McQuillan, D. C. Swan, S. S. Patel, and L. E. Markowitz, "Prevalence of HPV infection among females in the United States," J. Am. Med. Assoc. 297, 813-819 (2007).

22. J. V. Lacey, Jr., C. A. Swanson, L. A. Brinton, S. F. Altekruse, W. A. Barnes, P. E. Gravitt, M. D. Greenberg, O. C. Hadjimichael, L. McGowan, and R. Mortel, "Obesity as a potential risk factor for adenocarcinomas and squamous cell carcinomas of the uterine cervix," Cancer 98, 814-821 (2003).

23. E. M. Kanter, S. Majumder, G. J. Kanter, E. M. Woeste, and A. Mahadevan-Jansen, "Effect of hormonal variation on Raman spectra for cervical disease detection," Am. J. Obstet. Gynecol. 200, 512 (2009).

24. E. Vargis, E. M. Kanter, S. K. Majumder, M. D. Keller, R. B. Beaven, G. Rao, and A. Mahadevan-Jansen, "Effect of normal variations on disease classification of Raman spectra from cervical tissue," Analyst 139, 2981-2987 (2011).

25. M. S. Bergholt, W. Zheng, K. Lin, K. Y. Ho, M. Teh, K. G. Yeoh, J. B. So, and Z. Huang, "Characterizing variability in in vivo Raman spectra of different anatomical locations in the upper gastrointestinal tract toward cancer detection," J. Biomed. Opt. 16, 037003 (2011).

26. A. S. Haka, K. E. Shafer-Peltier, M. Fitzmaurice, J. Crowe, R. R. Dasari, and M. S. Feld, "Diagnosing breast cancer by using Raman spectroscopy," Proc. Nat. Acad. Sci. U. S. A. 102, 12371-12376 (2005).

27. L. Chrit, P. Bastien, G. D. Sockalingum, D. Batisse, F. Leroy, M. Manfait, and C. Hadjur, "An in vivo randomized study of human skin moisturization by a new confocal Raman fiber-optic microprobe: assessment of a glycerol-based hydration cream," Skin Pharmacol. Physiol. 19, 207-215 (2006) 
28. A. Cano, V. Serra, J. Rivera, R. Monmeneu, and C. Marzo, "Expression of estrogen receptors, progesterone receptors, and an estrogen receptorassociated protein in the human cervix during the menstrual cycle and menopause," Fertil. Steril. 54, 1058-1064 (1990).

29. S. F. Gould, J. M. Shannon, and G. R. Cunha, "The autoradiographic demonstration of estrogen binding in normal human cervix and vagina during the menstrual cycle, pregnacy, and the menopause," Am. J. Anat. 168, 229-238 (1983).

30. S. Thomsen and D. Tatman, "Physiological and pathological factors of human breast disease that can influence optical diagnosis," Ann. N.Y. Acad. Sci. 838, 171-193 (1998).

31. H. E. Nieburgs, "Recent progress in the interpretation of malignancy associated changes (MAC)," Acta Cytol. 12(6), 445-453 (1968).

32. J. B. Mitchell and L. A. McCormack, "Time trends in late-stage diagnosis of cervical cancer: differences by race/ethnicity and income," Med. Care 35, 1220-1224 (1997).

33. A. Jemal, R. Siegel, J. Xu, and E. Ward, "Cancer statistics, 2010," CA: Cancer J. Clinicians 60(5), 277-300 (2010).

34. E. Ward, A. Jemal, V. Cokkinides, G. K. Singh, C. Cardinez, A. Ghafoor, and M. Thun, "Cancer disparities by race/ethnicity and socioeconomic status," CA: Cancer J. Clinicians 54, 78-93 (2004).

35. J. Ferlay, H. R. Shin, F. Bray, D. Forman, C. Mathers, and D. M. Parkin, "Estimates of worldwide burden of cancer in 2008: GLOBOCAN 2008," Int. J. Cancer 127, 2893-2917 (2010).

36. E. E. Calle and R. Kaaks, "Overweight, obesity and cancer: epidemiological evidence and proposed mechanisms," Nat. Rev. Cancer 4, 579591 (2004).

37. T. J. Key, N. E. Allen, P. K. Verkasalo, and E. Banks, "Energy balance and cancer: the role of sex hormones," Proc. Nutr. Soc. 60, 81-89 (2001).

38. C. Macaulay, R. Richards-Kortum, U. Utzinger, A. Fedyk, E. Atkinson, D. Cox, and M. Follen, "Variation of fluorescence spectroscopy during the menstrual cycle," Opt. Express 10(12), 493-504 (2002).

39. K. C. Sung, M. Y. Dawood, S. Gregg, U. Urs, E. N. Atkinson, R. RK. Rebecca, and F. Michele, "Fluorescence spectroscopy for cervical precancer detection: Is there variance across the menstrual cycle?" $J$. Biomed. Opt. 7, 595-602 (2002).

40. R. Cubeddu, C. D'Andrea, A. Pifferi, P. Taroni, A. Torricelli, and G. Valentini, "Effects of the menstrual cycle on the red and near-infrared optical properties of the human breast," Photochem. Photobiol. 72, 383-391 (2000).

41. J. A. Knight, K. M. Blackmore, J. Wong, S. Tharmalingam, and L. Lilge, "Optical spectroscopy of the breast in premenopausal women reveals tissue variation with changes in age and parity," Med. Phys. 37, 419-426 (2010).

42. V. T. Chang, P. S. Cartwright, S. M. Bean, G. M. Palmer, R. C. Bentley, and N. Ramanujam, "Quantitative physiology of the precancerous cervix in vivo through optical spectroscopy," Neoplasia 11(4), 325-332 (2009).

43. J. G. Kelly, K. T. Cheung, C. Martin, J. J. O’Leary, W. Prendiville, P. L. Martin-Hirsch, and F. L. Martin, "A spectral phenotype of oncogenic human papillomavirus-infected exfoliative cervical cytology distinguishes women based on age," Clin. Chim. Acta 411, 1027-1033 (2010).
44. "Physical status: the use and interpretation of anthropometry," Report of a WHO Expert Committee," World Health Organ Tech. Rep. Ser. 854, Benteli, Swizterland (1995).

45. C. A. Lieber and A. Mahadevan-Jansen, "Automated method for subtraction of fluorescence from biological Raman spectra," Appl. Spectrosc. 57, 1363-1367 (2003).

46. B. Krishnapuram, L. Carin, M. A. Figueiredo, and A. J. Hartemink, "Sparse multinomial logistic regression: fast algorithms and generalization bounds," IEEE Trans. Pattern Anal. Mach. Intell. 27, 957-968 (2005).

47. G. J. Puppels, J. H. Olminkhof, G. M. Segers-Nolten, C. Otto, F. F. de Mul, and J. Greve, "Laser irradiation and Raman spectroscopy of single living cells and chromosomes: sample degradation occurs with $514.5 \mathrm{~nm}$ but not with $660 \mathrm{~nm}$ laser light," Exp. Cell Res. 195, 361-367 (1991).

48. C. A. Lieber, K. Molpus, K. Brader, and A. Mahadevan-Jansen, "Diagnostic tool for early detection of ovarian cancers using Raman spectroscopy," Proc. SPIE 3918, 129-130 (2000).

49. A. Mahadevan-Jansen and R. R. Richards-Kortum, "Raman spectroscopy for the detection of cancers and precancers," J. Biomed. Opt. 1, 31-70 (1996).

50. J. W. Tukey, Exploratory data analysis, Addison-Wesley, Reading, MA (1977).

51. R. J. Erckens, M. Motamedi, W. F. March, and J. P. Wicksted, "Raman spectroscopy for non invasive characterization of ocular tissue: potential for detection of biological molecules," J. Raman Spectrosc. 28, 293-299 (1997).

52. S. Fendel and B. Schrader, "Investigation of skin and skin lesions by NIR-FT-Raman spectroscopy," Fresenius' J. Anal. Chem. 360, 609-613 (1998).

53. U. Utzinger, D. L. Heintzelman, A. Mahadevan-Jansen, A. Malpica, M. Follen, and R. Richards-Kortum, "Near-infrared Raman spectroscopy for in vivo detection of cervical precancers," Appl. Spectrosc. 55, 955959 (2001).

54. C. J. Frank, D. C. Redd, T. S. Gansler, and R. L. McCreery, "Characterization of human breast biopsy specimens with near-IR Raman spectroscopy," Anal. Chem. 66, 319-326 (1994).

55. T. Miura and G. J. Thomas, Jr., "Raman spectroscopy of proteins and their assemblies," Subcell Biochem. 24, 55-99 (1995).

56. C. H. Liu, B. B. Das, W. L. Sha Glassman, G. C. Tang, K. M. Yoo, H. R. Zhu, D. L. Akins, S. S. Lubicz, J. Cleary, R. Prudente, E. Celmer, A. Caron, and R. R. Alfano, "Raman, fluorescence, and time-resolved light scattering as optical diagnostic techniques to separate diseased and normal biomedical media," J. Photochem. Photobiol. B 16, 187209 (1992).

57. D. N. Danforth, A. Veis, M. Breen, H. G. Weinstein, J. C. Buckingham, and P. Manalo, "The effect of pregnancy and labor on the human cervix: changes in collagen, glycoproteins, and glycosaminoglycans," Am. J. Obstet. Gynecol. 120, 641-651 (1974).

58. S. Y. Yu, C. A. Tozzi, J. Babiarz, and P. C. Leppert, "Collagen changes in rat cervix in pregnancy-polarized light microscopic and electron microscopic studies," Proc. Soc. Exp. Biol. Med. 209(4), 360-368 (1995).

59. K. J. Rothman, "BMI-related errors in the measurement of obesity," Int. J. Obes. 32, S56-S59 (2008). 